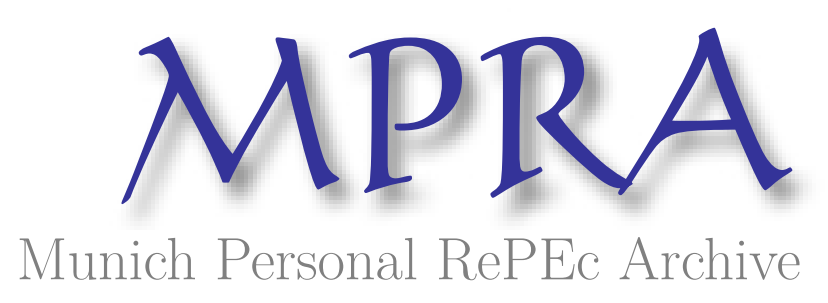

\title{
Multivariate bubbles and antibubbles
}

Fry, John

University of Sheffield

19 May 2014

Online at https://mpra.ub.uni-muenchen.de/56081/

MPRA Paper No. 56081, posted 19 May 2014 18:02 UTC 


\title{
Multivariate bubbles and antibubbles
}

\author{
John Fry*
}

May 2014

\begin{abstract}
In this paper we develop models for multivariate financial bubbles and antibubbles based on statistical physics. In particular, we extend a rich set of univariate models to higher dimensions. Changes in market regime can be explicitly shown to represent a phase transition from random to deterministic behaviour in prices. Moreover, our multivariate models are able to capture some of the contagious effects that occur during such episodes. We are able to show that declining lending quality helped fuel a bubble in the US stock market prior to 2008. Further, our approach offers interesting insights into the spatial development of UK house prices.
\end{abstract}

\section{Introduction}

The analogy between financial crashes and phase transitions in critical phenomena in statistical physics is now well established [1]-[2] and a large literature discusses the subject of log-periodic precursors to financial crashes - see e.g. [3]-[10]. For a review see [11]-[12]. Despite their origins in statistical physics log-periodic models have begun to appear in the mainstream finance literature [12], [13]-[16]. Thus, having achieved an element of wider significance the subject is simply too important to ignore.

Financial markets operate by balancing risk and return [17]. As discussed in [18]-[19] there is a sense in which the prevailing class of log-periodic models omits a crucial second-order related to market over-confidence. There is thus an interesting sense in which the academic literature reflects wider market failings prior to the 2008 crisis [20]. Here, a better physical model leads to a more elegant approach - one that in turn can be easily extended to higher dimensions. In particular, in a multivariate setting we can show how correlation in the bubble/antibubble process feeds through into observed prices.

Bubbles and anti-bubbles [21] are a core theme explored by log-periodic and related models although a wide range of alternative applications are possible [19]. Multivariate bubbles have not been widely studied and the area appears much under-explored. The ability to fit multivariate bubble and antibubble models to data is significant and allows for a more systematic approach in empirical applications. Multivariate models allow for the simultaneous tracking of multiple markets. This is important as previous work has often studied different types of financial markets [22]-[24] or multiple regional markets [25]. Multivariate models also allow us to study contagion [26]-[27]. Our model is inherently practical in nature. In addition to the above our approach also allows for empirical tests for bubbles and antibubbles and can also allow us to provide empirical estimates for the level of over-pricing and the level of under-pricing.

${ }^{*}$ The University of Sheffield, Management School, Conduit Road, Sheffield S10 1FL, UK. E-mail: John.Fry@sheffield.ac.uk 
The empirical analyses in this paper are interesting and important in their own right. Firstly, we are able to show that declining credit quality helped fuel a bubble in the US stock market. Secondly, we apply our model to English house prices. Both applications and clear ramifications for recent and on-going crises as the economic impact of house-price crashes can be particularly severe [28]-[29]. In modelling the contagion in English house prices we can show that the much-heralded North-South divide is indeed pronounced and may be under-stated by conventional economic approaches [30]. English house prices appear to be dominated by the South Eastern corner of the country (London, South East, Metropolitan and East Anglia regions) with comparatively little evidence for contagion between neighbouring geographic regions.

The layout of this paper is as follows. Section 2 introduces a univariate model for bubbles and antibubbles. This is then extended to multivariate and bivariate settings in Section 3 . Empirical applications are discussed in Section 4. Section 5 concludes.

\section{A univariate bubble model}

Markets work by balancing the level of risk and the rate of return. The level of risk and return remain stable even in the face of technological innovation or an influx of new investors [31]. These assumptions do not rely on complicated mathematics and avoid dubious assumptions such as the "riskless hedge" of the Black-Scholes model [32]. Our model makes several observable predictions for market crashes. Inter alia speculation-induced crashes are preceded by an unsustainable super-exponential growth coupled with a detectable increase in market over-confidence.

Let $P_{t}$ denote the price of an asset at time $t$ and let $X_{t}=\log P_{t}$. The set up of the model is as follows:

Assumption 1 (Intrinsic Rate of Return) The intrinsic rate of return is assumed constant and equal to $\mu$ :

$$
E\left[X_{t+\Delta}-X_{t} \mid X_{t}\right]=\mu \Delta+o(\Delta)
$$

Assumption 2 (Intrinsic Level of Risk) The intrinsic level of risk is assumed constant and equal to $\sigma^{2}$ :

$$
\operatorname{Var}\left[X_{t+\Delta}-X_{t} \mid X_{t}\right]=\sigma^{2} \Delta+o(\Delta)
$$

As in [1] our starting point is the equation

$$
P(t)=P_{1}(t)(1-\kappa)^{j(t)},
$$

where $P_{1}(t)$ satisfies

$$
d P_{1}(t)=\left[\mu(t)+\sigma^{2}(t) / 2\right] P_{1}(t) d t+\sigma(t) P_{1}(t) d W_{t},
$$

where $W_{t}$ is a Wiener process and $j(t)$ is a jump process satisfying

$$
j(t)=\left\{\begin{array}{cc}
0 & \text { before the crash } \\
1 & \text { after the crash. }
\end{array}\right.
$$

When a crash occurs $\kappa \%$ is automatically wiped off the value of the asset. Prior to a crash $P(t)=P_{1}(t)$ and $X_{t}=\log (P(t))$ satisfies

$$
d X_{t}=\mu(t) d t+\sigma(t) d W_{t}-v d j(t),
$$


where $v=-\ln [(1-\kappa)]>0 .^{*}$ Assumptions 1-2 show that crashes are outliers and can, in principle, be predicted based on anomalous behaviour in the drift and volatility in equation (6). In a bubble regime a representative investor is compensated for the crash risk by an increased rate of return with $\mu(t)>\mu$ the long-term rate of return. This is accompanied by a decrease in the volatility function $\sigma^{2}(t)$ - a result which at first glance may appear counter-intuitive but, in fact, represents market over-confidence [18]-[19].

Suppose that a crash has not occurred by time $t$. In this case we have that

$$
\begin{aligned}
E[j(t+\Delta)-j(t)] & =\Delta h(t)+o(\Delta), \\
\operatorname{Var}[j(t+\Delta)-j(t)] & =\Delta h(t)+o(\Delta),
\end{aligned}
$$

where $h(t)$ is the hazard rate. Hence it follows from (21) and (7) that

$$
\mu(t)-v h(t)=\mu ; \quad \mu(t)=\mu+v h(t) .
$$

Equation (9) thus returns the first-order model - namely that the rate of return must increase in order to compensate a representative investor for the risk of a crash.

Second-order condition. This condition stipulates that in order for a bubble to develop a rapid growth in prices is not sufficient in isolation. The perceived price risk must also diminish. From equations (2) and (8) it follows that

$$
\sigma^{2}(t)+v^{2} h(t)=\sigma^{2} ; \quad \sigma^{2}(t)=\sigma^{2}-v^{2} h(t) .
$$

Equation (10) thus describes a collective market over-confidence that arises as a result of the bubble and leads to an under-estimation of the true long-term level of volatility. We note that from a mathematical perspective equation (10) holds some wider significance [18] since it satisfies a phase-transition condition delineating between random and deterministic behaviour in prices:

$$
\min _{t} \sigma^{2}(t)=0 \text {. }
$$

Post-crash increase in volatility. Further to the above discussion equation (10) also predicts that volatility increases after the crash - in line with the predictions of several related models (see e.g. [34]). Before the market crashes, in the bubble regime, we have that

$$
\tilde{\sigma}^{2}=\operatorname{Var}\left(X_{t+\Delta} \mid X_{t}\right)=\Delta\left[\sigma^{2}-v^{2} h(t)\right]+o(\Delta),
$$

whilst after the crash

$$
\operatorname{Var}\left(X_{t+\Delta} \mid X_{t}\right)=\Delta\left[\tilde{\sigma}^{2}+v^{2} h(t)\right]+o(\Delta) .
$$

Equations (9-10) show that specification of the hazard function $h(t)$ completes the model. Equation (10) shows that an important feature of our model is that the hazard function remains bounded. This is in order to ensure that $\sigma^{2}(t)$ remains non-negative. With this in mind, and for computational reasons, we follow [18] in choosing

$$
h(t)=\frac{\beta t^{\beta-1}}{\alpha^{\beta}+t^{\beta}} .
$$

\footnotetext{
${ }^{*}$ In the sequel we note that the case of an antibubble is the same basic model but with $v$ replaced by $-v$ throughout [33].
} 
Equation (14) has fewer degrees of freedom than alternative specifications considered in [1] and in several subsequent papers. This choice of hazard function also matches related modelling and phenomenology of housing markets discussed in [35] which is of interest given the empirical application of our model to English house prices in Section 4. Equation (14) corresponds to a log-logistic model popular in mathematical statistics [36] which nevertheless captures the essential aspects of previous approaches; the hazard function has a non-trivial modal point at $t=\alpha(\beta-1)^{1 / \beta}$ with modal point $(\beta-1)^{1-1 / \beta} / \alpha$.

As laid out above, our model can be used to empirically test for the presence of bubbles in a given price series. However, the scope of our model extends further and also enables us to estimate the speculative bubble component present within observed prices. Under fundamental price dynamics with $v=0$

$$
P_{F}(t):=E(P(t))=P(0) e^{\tilde{\mu} t},
$$

where $\tilde{\mu}=\mu+\sigma^{2} / 2$. In empirical work we can use equation (15) to estimate fundamental value an approach which recreates the widespread phenomenology of approximate exponential growth in economic time series [37]. Define

$$
H(t):=\int_{0}^{t} h(u) d u
$$

Under a speculative bubble, with $v>0$, we have that

$$
X_{t} \sim N\left(X_{0}+\mu t+v H(t), \sigma^{2} t-v^{2} H(t)\right) .
$$

Hence, it follows from (17) that

$$
P_{B}(t):=E(P(t))=P(0) e^{\tilde{\mu} t+\left(v-\frac{v^{2}}{2}\right) H(t)} .
$$

Equations (15-18) lead to the following estimate of the speculative bubble component defined as the average distance between fundamental and bubble prices:

$$
\begin{aligned}
\text { Bubble Component } & =1-\frac{1}{T} \int_{0}^{T} \frac{P_{F}(t)}{P_{B}(t)} d t \\
& =1-\frac{1}{T} \int_{0}^{T}\left(1+\frac{t^{\beta}}{\alpha^{\beta}}\right)^{-\left(v-\frac{v^{2}}{2}\right)} d t .
\end{aligned}
$$

Given plug-in estimates of $\alpha, \beta$ and $v$ the integral in (19) can be calculated numerically. Equation (19) should result in a fraction in $(0,1)$. In [38] this gave a value of 0.202 for UK house prices over the years 2002-2007 suggesting that the bubble accounted for around $20 \%$ of observed prices - closely matching a subsequent fall in UK house prices of around 20\% in 2008-9.

An antibubble represents the mirror image of a speculative bubble [33]. Just as speculative bubbles result in dramatic price rises antibubbles can result in dramatic price falls. Antibubbles can be modelled by replacing $v$ with $-v$ in the above. In the case of an antibubble, analogous reasoning leads to an estimate of the level of under-pricing. Define

$$
P_{A B}(t):=E(P(t))=P(0) e^{\tilde{\mu} t-\left(v+\frac{v^{2}}{2}\right) H(t)}
$$


It follows that

$$
\begin{aligned}
\text { Antibubble Component } & =1-\frac{1}{T} \int_{0}^{T} \frac{P_{F}(t)}{P_{A B}(t)} d t \\
& =1-\frac{1}{T} \int_{0}^{T}\left(1+\frac{t^{\beta}}{\alpha^{\beta}}\right)^{\left(v+\frac{v^{2}}{2}\right)} d t .
\end{aligned}
$$

Similarly, $(20)$ should yield a fraction in $(-1,0)$. E.g. a value of -0.1 would suggest that prices are under-valued by roughly $10 \%$.

\section{The multivariate model}

In this subsection we discuss multivariate models for bubbles. Thus we are able to describe the price of more-than-one asset simultaneously. This is significant for empirical applications across different countries [26]-[27]. Even within the same country regional differences, in housing and other markets, can be pronounced.

Let $P_{t}$ denote the prices $\left(P_{t}^{1}, \ldots, P_{t}^{p}\right)$ of a basket of $p$ assets at time $t$. Define $X_{t}=$ $\left(X_{t}^{1}, \ldots, X_{t}^{p}\right)$ where $X_{t}^{i}=\log P_{t}^{i}$. For the multivariate model Assumptions 1 and 2 are replaced by their vector/matrix analogues.

Assumption 1: [Intrinsic Rate of Return] The intrinsic rate of return is assumed constant and equal to $\mu$ :

$$
E\left[X_{t+\Delta}-X_{t} \mid X_{t}\right]=\mu \Delta+o(\Delta)
$$

Assumption 2: [Intrinsic Level of Risk] The intrinsic level of risk is assumed constant and equal to $\Sigma$ :

$$
\operatorname{Var}\left[X_{t+\Delta}-X_{t} \mid X_{t}\right]=\Sigma \Delta+o(\Delta) .
$$

Co-ordinatewise our starting equation (3) becomes

$$
p^{i}(t)=p_{1}^{i}(t)\left(1-\kappa^{i}\right) j(t)
$$

and before the crash $X_{t}$ satisfies the vector-valued equation

$$
d X_{t}=\mu(t) d t+\sqrt{\sigma(t)} d W_{t}-v d j(t),
$$

where $v$ is the diagonal matrix satisfying $v_{i i}=-\ln \left(1-\kappa_{i}\right)=v_{i}$. Assumption 1 above yields a vector-valued re-statement of equation (9):

$$
\mu(t)-v h(t)=\mu ; \mu(t)=\mu+v h(t) .
$$

Similarly, Assumption 2 shows that the second-order condition now becomes

$$
\Sigma(t)+v \Sigma_{j} v^{T} h(t)=\Sigma ; \Sigma(t)=\Sigma-v \Sigma_{j} v^{T} h(t) .
$$

where $\Sigma_{j}$ denotes the correlation matrix of $j(t)$. Equation (26) thus shows how correlation in the bubble process is transferred to prices prior to the crash. Genuinely high-dimensional and multivariate models are possible though it seems that these may lose some interpretability. Since bivariate models are by far the most convenient and natural to use in applications in the sequel we restrict to a bivariate model. 


\subsection{A bivariate bubble model}

In a bivariate extension of the preceding univariate and multivariate models equation (24) becomes

$$
d X_{t}=\mu(t) d t+\sqrt{\Sigma(t)} d W_{t}-v d j(t)
$$

where $X_{t}=\left(X_{1}(t), X_{2}(t)\right)^{T}$ denotes the log-price of Assets 1 and 2 at time $t, \Sigma(t)$ is the instantaneous covariance and $W_{t}$ is standard bivariate Brownian motion. Assumption 1 gives

$$
\mu_{1}(t)=\mu_{1}+v_{1} h(t) ; \quad \mu_{2}(t)=\mu_{2}+v_{2} h(t) .
$$

Assumption 2 gives

$$
\begin{aligned}
\Sigma(t) & =\left(\begin{array}{cc}
\sigma_{1}^{2} & \sigma_{12} \\
\sigma_{12} & \sigma_{2}^{2}
\end{array}\right)-\left(\begin{array}{cc}
v_{1} & 0 \\
0 & v_{2}
\end{array}\right)\left(\begin{array}{cc}
1 & \rho \\
\rho & 1
\end{array}\right)\left(\begin{array}{cc}
v_{1} & 0 \\
0 & v_{2}
\end{array}\right) h(t), \\
& =\left(\begin{array}{cc}
\sigma_{1}^{2} & \sigma_{12} \\
\sigma_{12} & \sigma_{2}^{2}
\end{array}\right)-\left(\begin{array}{cc}
v_{1}^{2} & \rho v_{1} v_{2} \\
\rho v_{1} v_{2} & v_{1}^{2}
\end{array}\right) h(t) .
\end{aligned}
$$

In addition to equation (10) the phase-transition condition also gives

$$
\min _{t} \Sigma(t)=0 ; \min _{t} \sigma_{12}-\rho v_{1} v_{2} h(t)=0 .
$$

Historical Estimation Bias. Equation (30) when taken together with equations (9-10) serve to highlight possible dangers regarding historical estimation bias - an issue with specific relevance to the CDO crisis (see e.g. [39]). We have already seen that during a bubble regime prices may be rising at artificially high rates with comparatively little volatility compared to the underlying long-term values. Equation (30) is also useful in highlighting that using historical prices in a bubble regime may lead to under-diversified portfolios as a consequence of under-estimating long-term correlation levels in returns series. If a crash occurs at time $t_{0}$, in addition to an increase in marginal volatility, the covariance of $\Delta X_{1}\left(t_{0}\right)$ and $\Delta X_{2}\left(t_{0}\right)$ increases by a factor of $\rho v_{1} v_{2} h\left(t_{0}\right)$ (from $\sigma_{12}-\rho v_{1} v_{2} h\left(t_{0}\right)$ to its equilibrium value of $\sigma_{12}$ ).

Contagion. The above discussion leads naturally to an empirical test for contagious effects that arise as part of the bubble process. As discussed below this involves testing the hypothesis shown in equation (35). Suppose we have two assets whose prices are given by $e^{X(t)}$ and $e^{Y(t)}$. Let $\Delta X_{t}=X_{t+1}-X_{t}$. Under the model (27) knowledge of $Y(t)$ reduces uncertainty in $X(t)$ by

$$
\begin{aligned}
\operatorname{Var}[\Delta X(t)]-\operatorname{Var}[\Delta X(t) \mid \Delta Y(t)] & =\operatorname{Var}\left[\Delta X_{t}\right]-\left(1-\operatorname{Cor}^{2}\left(\Delta X_{t}, \Delta Y_{t}\right)\right) \operatorname{Var}\left[\Delta X_{t}\right] \\
& =\operatorname{Cor}^{2}\left(\Delta X_{t}, \Delta Y_{t}\right) \operatorname{Var}\left[\Delta X_{t}\right] .
\end{aligned}
$$

Similarly, knowledge of $X(t)$ reduces uncertainty in $Y(t)$ by the amount

$$
\operatorname{Var}[\Delta Y(t)]-\operatorname{Var}[\Delta Y(t) \mid \Delta X(t)]=\operatorname{Cor}^{2}\left(\Delta X_{t}, \Delta Y_{t}\right) \operatorname{Var}\left[\Delta Y_{t}\right]
$$

The constraints $\sigma_{X}^{2}(t) \geq 0$ and $\sigma_{Y}^{2}(t) \geq 0$ imply that

$$
\sigma_{X}^{2}=\frac{v_{X}^{2}(\beta-1)^{1-\frac{1}{\beta}}}{\alpha} ; \sigma_{Y}^{2}=\frac{v_{Y}^{2}(\beta-1)^{1-\frac{1}{\beta}}}{\alpha} .
$$


Contagion from $Y(t)$ to $X(t)$ occurs if $Y(t)$ is more informative about $X(t)$ than $X(t)$ is about $Y(t)$. From equations (32-33) contagion from $Y(t)$ to $X(t)$ occurs if

$$
\begin{aligned}
\operatorname{Cor}^{2}\left(\Delta X_{t}, \Delta Y_{t}\right) \operatorname{Var}\left[\Delta X_{t}\right] & <\operatorname{Cor}^{2}\left(\Delta X_{t}, \Delta Y_{t}\right) \operatorname{Var}\left[\Delta Y_{t}\right] \\
\operatorname{Var}\left[\Delta X_{t}\right] & <\operatorname{Var}\left[\Delta Y_{t}\right] \\
v_{X}^{2}\left[\frac{(\beta-1)^{1-\frac{1}{\beta}}}{\alpha}-\ln \left(\frac{\alpha^{\beta}+(t+1)^{\beta}}{\alpha^{\beta}+t^{\beta}}\right)\right] & <v_{Y}^{2}\left[\frac{(\beta-1)^{1-\frac{1}{\beta}}}{\alpha}-\ln \left(\frac{\alpha^{\beta}+(t+1)^{\beta}}{\alpha^{\beta}+t^{\beta}}\right)\right] \\
v_{X}^{2} & <v_{Y}^{2} .
\end{aligned}
$$

Equation (35) is significant as it shows that contagion occurs as the overall bubble process becomes dominated by price rises and speculation in Asset $Y$. Similarly in an antibubble contagion from $Y(t)$ to $X(t)$ occurs as speculation that drives down the price of $Y(t)$ becomes the dominant effect.

\section{Empirical applications}

\subsection{Multivariate bubbles}

We illustrate our multivariate bubble models with an application to a data set consisting of the S\& P 500 and the Federal Funds Rate (FFR). The joint behaviour of US interest rates is much studied [22]-[24] and is also of wider interest amid concern that loose US monetary policy has inflated a succession of recent bubbles [24].

The FFR is the interest rate at which depositing institutions actively trade balances held at the Federal Reserve. In particular, data published as the FFR effective rate represents the weighted averaged across all such transactions. As the rate increases it becomes more expensive for financial institutions to borrow funds. One feature of interest is whether or not the FFR increases as a symptom of wider problems with credit worthiness. In a similar vein to the original model in [1] increases in the FFR may compensate lending institutions for the Credit Risk that they bear. It is well known that such structural problems and antibubbles in the underlying can lead to dramatic increases and bubbles in the associated interest rates [19] - see the Appendix for further details.

Following a similar approach in [23] we analyse weekly data from January 2003 to June 2007. A plot of the S\& P 500 and the FFR is shown below in Figure 1. Both series show a rapid growth over time consistent with earlier suggestions of a bubble in both series. Results in Table 1 give conclusive evidence of a bubble in both univariate series. This is subsequently confirmed by the bivariate bubble model in Section 2.2. Further, the test for contagion in equation (35) suggests evidence for contagion running from the FFR to the S\& P 500. This would appear to confirm similar findings of debt-fuelled bubbles in [24]. In the lead in to the crisis the FFR increased as a symptom of generally decreasing credit quality in the wider financial system. This then spilled over and led to an unsustainable bubble in the US stock market.

\subsection{Multivariate antibubbles}

We illustrate our multivariate models for antibubbles with an application to novel data on English house prices. The data consists of house prices for 10 English regions obtained from the Nationwide building society ${ }^{\dagger}$. Nominal house prices are then re-scaled by a GDP deflator in

\footnotetext{
${ }^{\dagger}$ http://www.nationwide.co.uk/hpi/datadownload/data_download.htm
} 


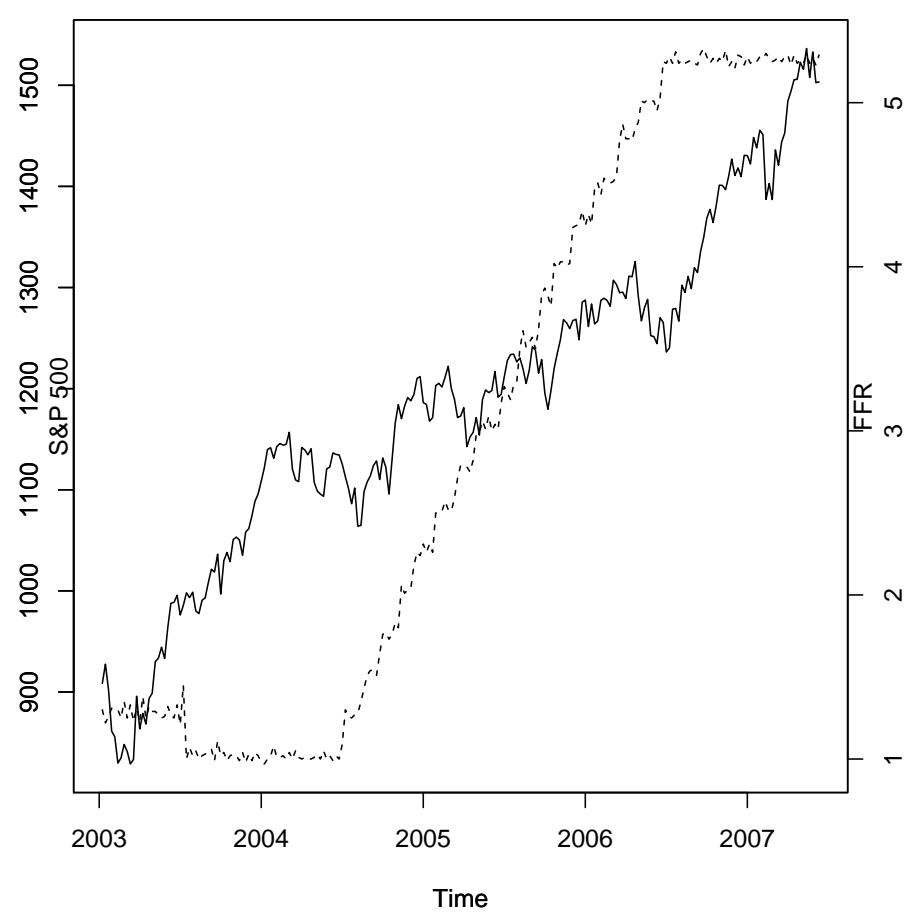

Figure 1: S\& P 500 (solid lines) and Federal Funds Rate (FFR) (dashed lines).

order to translate nominal prices into real prices.

A plot of UK house prices over the period in question is shown below in Figure 2 and suggests that the antibubble that started in 2008 has been particularly severe - especially once we adjust house prices for inflation. Results shown in Table 2 below give strong statistical evidence for an antibubble in each of the ten English regions. Table 3 shows the estimated size of the antibubbles. In each region statistical evidence of an antibubble is accompanied by a significant economic effect. The impact of the antibubble splits neatly along the north-south divide - although results suggest that the East Midlands region, commonly identified as being in the South of England economically [30], should in fact be seen as part of the North of England. In the South of England house prices seem to have falled by around 1/3 in real terms. In the North of England this figure appears closer to 20\%.

Generally, the economy of the South of England over-shadows that of the North [30]. Results in Table 4 show that English house prices tend to be dominated by the South Eastern corner of England. Price falls in the South of England spillover and infect the North. Results in Table 4 give evidence for contagion running from London, Metropolitan, South East and East Anglia to the East and West Midlands.Contagion is also found running from London to the North and North West, from the South East to the North and from East Anglia to the North. Results also appear consistent with evidence for a preceding housing bubble in UK house prices circa 19992006 due in large part to speculation in house prices in the South of England [19]. In contrast, there appears to be relatively limited evidence of contagion across neighbouring English regions. This is not say that prices in neighbouring regions are independent. Generally, what have in 


\begin{tabular}{l|l|l|}
\hline \hline \multicolumn{3}{c}{ Univariate bubble model } \\
\hline \hline & $t$-value & $p$-value \\
\hline \hline S\& P 500 & 162101.6 & 0.000 \\
FFR & 8.306 & 0.000 \\
\hline \multicolumn{3}{|c|}{ Test for contagion } \\
\hline \hline & $t$-value & $p$-value \\
\hline \hline & 22.896 & 0.000 \\
\hline \hline
\end{tabular}

Table 1: Results for the statistical tests for bubbles

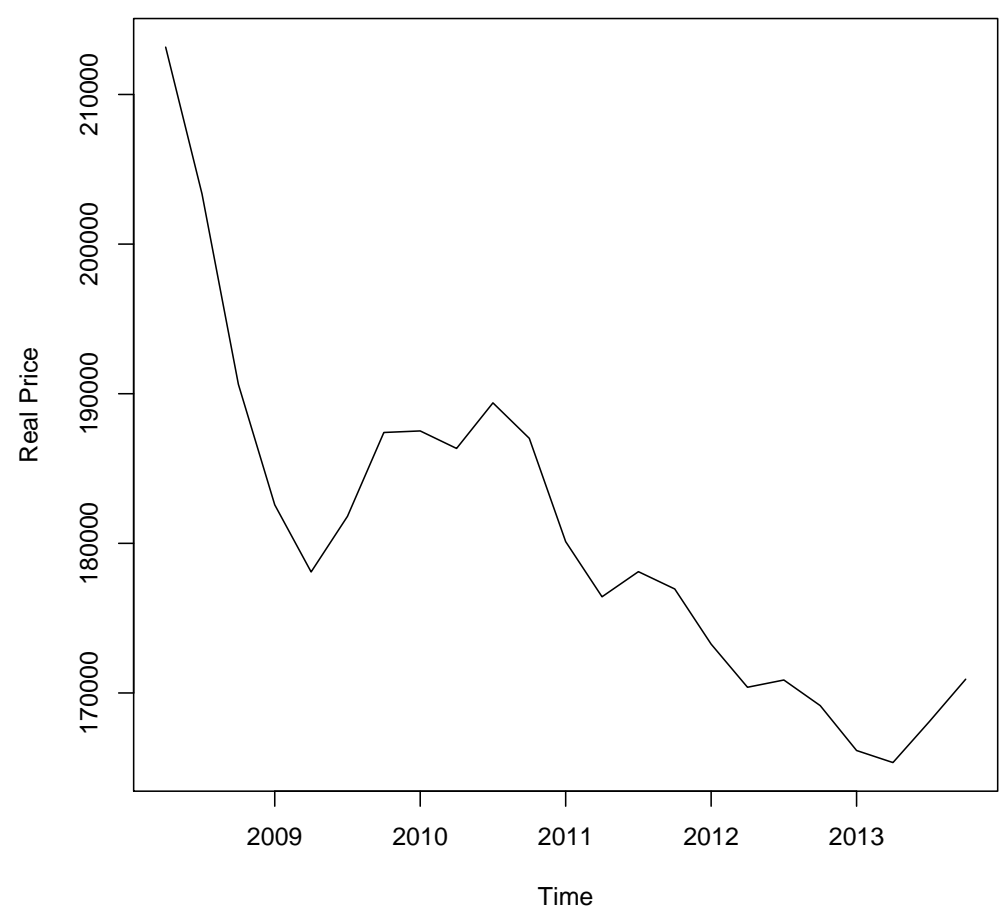

Figure 2: English house prices 2008-2013

these cases is co-dependence rather than an asymmetric contagious effect.

\section{Conclusions and further work}

This paper has provided simple mathematical models for multivariate bubbles and antibubbles adding to several recent developments in the area [18]-[19]. Bubbles occur as the price rises to compensate a representative investor for the risk of a crash. Similarly, antibubbles occur as the price decreases as compensation for the risk associated with a subsequent market correction. This is accompanied in our second-order model by a detectable increase in market over-confidence 


\begin{tabular}{|l|l|l|}
\hline Region & $t$-value & $p$-value \\
\hline South West & 11.245 & 0.000 \\
London & 6.975 & 0.000 \\
Metropolitan & 11.683 & 0.000 \\
South East & 9.048 & 0.000 \\
East Anglia & 7.726 & 0.000 \\
West Midlands & 8.348 & 0.000 \\
East Midlands & 8.597 & 0.000 \\
North West & 6.954 & 0.000 \\
Yorkshire & 5.152 & 0.000 \\
North & 10.066 & 0.000 \\
\hline
\end{tabular}

Table 2: $P$-values testing the null hypotheses of no anti-bubble 2008-13

\begin{tabular}{|l|l|}
\hline Region & Estimated anti-bubble component \\
\hline South West & -0.334 \\
London & -0.322 \\
Metropolitan & -0.370 \\
South East & -0.334 \\
East Anglia & -0.336 \\
West Midlands & -0.232 \\
East Midlands & -0.262 \\
North West & -0.208 \\
Yorkshire & -0.214 \\
North & -0.238 \\
\hline
\end{tabular}

Table 3: Estimated size of antibubbles by English region 2008-2013

\begin{tabular}{|l|l|l|l|l|l|l|l|l|l|l|}
\hline & SW & L & M & SE & EA & WM & EM & NW & Y & N \\
\hline SW & $\cdot$ & 0.404 & 0.513 & 0.543 & 0.433 & 0.058 & 0.193 & 0.168 & 0.536 & 0.055 \\
\hline L & 0.404 & $\cdot$ & 0.422 & 0.394 & 0.853 & $0.020 *$ & $0.046 *$ & $0.037 *$ & 0.076 & $0.048 *$ \\
\hline M & 0.513 & 0.422 & $\cdot$ & 0.891 & 0.564 & $0.019 *$ & $0.046 *$ & 0.091 & 0.265 & 0.078 \\
\hline SE & 0.543 & 0.394 & 0.891 & $\cdot$ & 0.581 & $0.025 *$ & $0.035 *$ & 0.078 & 0.209 & $0.045 *$ \\
\hline EA & 0.433 & 0.853 & 0.564 & 0.581 & $\cdot$ & $0.028 *$ & $0.032 *$ & 0.080 & 0.195 & $0.048 *$ \\
\hline WM & 0.058 & $0.020 *$ & $0.019 *$ & $0.025 *$ & $0.028 *$ & $\cdot$ & 0.899 & 0.781 & 0.227 & 0.825 \\
\hline EM & 0.193 & $0.046 *$ & $0.046 *$ & $0.035 *$ & $0.032 *$ & 0.899 & $\cdot$ & 0.870 & 0.427 & 0.630 \\
\hline NW & 0.168 & $0.037 *$ & 0.091 & 0.078 & 0.080 & 0.781 & 0.870 & $\cdot$ & 0.359 & 0.633 \\
\hline Y & 0.536 & 0.076 & 0.265 & 0.209 & 0.195 & 0.227 & 0.427 & 0.359 & $\cdot$ & 0.285 \\
\hline N & 0.055 & $0.048 *$ & 0.078 & $0.045 *$ & $0.048 *$ & 0.825 & 0.630 & 0.633 & 0.285 & $\cdot$ \\
\hline
\end{tabular}

Table 4: $P$-values testing the null hypotheses of no pairwise contagion during the English housing anti-bubble 2008-13 
akin to phase-transition behaviour in statistical physics [40]. Our model highlights a possible issue with historical estimation bias. Relying on historical prices only may over-estimate gains (losses) during a bubble (antibubble), may under-estimate the true level of long-term risk and may also under-estimate long-term correlation levels potentially leading to under-diversified portfolios.

Our multivaraite models allow for a more systematic approach in empirical applications such as comparing multiple markets and evaluating contagion. Here, we can show that this leads to empirical applications that are interesting and important in their own right. The interplay between stock markets and the Federal Funds Rate (FFR) is interesting and important [22]-[24]. Firstly, we are able to show that a bubble in the FFR spills over and infects the US stock market prior to the crash of 2008. This appears to tie in closely with recent suggestions that Federal Reserve policy was directly responsible as a bubble inflated against the backdrop of decreasing lending quality [24]. Secondly, we look at the English housing antibubble that occurred from 2008-13. Evidence is found that price falls in the South Eastern part of the country "infect" the North. The often talked about North-South divide is clearly evident in English house prices. Further, results suggest that the scale of the division has been under-stated by some conventional economic approaches [30]. Allied to the above there appears to be relatively little evidence of contagion across neighbouring regions.

The bubble and antibubble models discussed in this paper are potentially very rich. Additional applications include financial aspects of societal resilience [41], economic policy [42] and market psychology and trading [43]. Future work will also explore additional links with the model in [31].

\section{Appendix: Antibubble-generated bubbles in bond yields}

It is easy to show that an antibubble in the price of the underlying asset leads to a bubble in the corresponding Bond yields [19]. Following the standard approach [44] write

$$
P(t)=M e^{-y(t) T},
$$

where $y(t)$ is the yield, $T$ is the maturity date, $M$ is the constant value of the bond at maturity and $P(t)$ is the price of the underlying asset. It follows that $X(t)=\ln P(t)$ satisfies

$$
X(t)=\ln M-y(t) T .
$$

Under the equation for an antibubble we have that

$$
d X_{t}=\mu(t) d t+\sigma(t) d W_{t}+v d j(t),
$$

where

$$
\begin{aligned}
\mu(t) & =\mu-v h(t), \\
\sigma^{2}(t) & =\sigma^{2}-v^{2} h(t) .
\end{aligned}
$$

Combining equations (37-39) it follows that the bond yields $y(t)$ satisfy

$$
d y(t)=-\frac{\mu(t)}{T} d t+\frac{\sigma(t)}{T} d W_{t}^{\prime}-\frac{v}{T} d j(t),
$$

where $W_{t}^{\prime}=-W_{t}$. Thus it follows that (40) gives the formula for a speculative bubble since $W_{t}^{\prime} \stackrel{d}{=} W_{t}$. 


\section{References}

[1] A. Johansen, O. Ledoit, and D. Sornette, I. J. Theor. and Appl. Financ., 3, 219 (2000)

[2] D. Sornette. Why stock markets crash: critical events in complex financial systems. (Princeton University Press, Princeton, 2003)

[3] W-X. Zhou and D. Sornette, Phys. A. 387, 243 (2008).

[4] W-X. Zhou and D. Sornette, Phys. A. 388, 869 (2009).

[5] P., Sieczka, D., Sornette, and J. A. Holyst, Eur. Phys. J. B 82, 257 (2011)

[6] J. A. Feigenbaum, Quant. Financ. 1, 346 (2001)

[7] J. Feigenbaum, Quant. Financ. 1, 527 (2001)

[8] G. Chang and J. Feigenbaum, Quant. Financ. 6, 15 (2006)

[9] G. Chang and J. Feigenbaum, Quant. Financ. 8, 723 (2008)

[10] D. Bree, D. Challet, D. and P.P. Perrano, Quant. Financ. 13, 275 (2013)

[11] D. Sornette, R. Woodard, W. Yan and W-X. Zhou, Physica A 392, 4417 (2013)

[12] P. Geraskin and D. Fantazzini, Eur. J. of Financ. 19, 366 (2013)

[13] L. Lin and D. Sornette, Eur. J. of Financ. 19, 344 (2013)

[14] J. R. Kurz-Kim, Applied Econ. Lett. 19, 1465 (2012)

[15] Z-Q. Jiang, W-X. Zhou, D. Sornette, R. Woodard, K. Bastiaensen and P. Cauwels, J. of Econ. Behav. and Organ. 74, 149 (2010)

[16] D. Bree and N. Joseph, Int. Rev. of Financ. Anal. 30, 287 (2013)

[17] H.M. Markowitz, Portfolio Selection: Efficient Diversification of Investments, 2nd edn. (Blackwell, Malden, Massachussets, 1971).

[18] J. Fry, Eur. Phys. J. B 85, 405 (2012).

[19] J. Fry, Eur. Phys. J. B 87, 1 (2014).

[20] R. Peston and L. Knight, How do we fix this mess? The economic price of having it all and the route to lasting prosperity, (Hodder and Stoughton, London, 2012)

[21] W-X. Zhou and D. Sornette, Phys. A 348, 428 (2005)

[22] W-X. Zhou and D. Sornette, Phys. A 337, 586 (2004)

[23] K. Guo, W-X. Zhou, S-W. Cheng, and D. Sornette, PLoS One 6, e22794 (2011)

[24] D. Sornette and P. Cauwels, Risks 2, 103 (2014).

[25] D. Ardila, P. Cauwels, D. Sanadgol and D. Sornette, The Swiss Real Estate J. 6, 38 (2014) 
[26] D. Sornette and Y. Malevergne, Extreme financial risks: From dependence to risk management. (Springer, Berlin Heidelberg New York, 2006).

[27] A. McNeil, R. Frey and P. Embrechts, Quantitative risk management. (Princeton University Press, Princeton, 2005).

[28] C. Hott and P. Monnin, J. of Real Estate and Financ. Econ. 36, 427 (2008)

[29] A. Black, P. Fraser and M. Hoesli, J. of Bus. Financ. and Account. 33, 1535 (2006)

[30] R. Rowthorn, Spat. Econ. Anal., 5, 363 (2010)

[31] J. Zeira, J. of Monet. Econ. 43, 237 (1999)

[32] J-P. Bouchaud, and M. Potters, Theory of financial risk and derivative pricing. From statistical physics to risk management, 2nd edn. (Cambridge University Press, Cambridge, 2003).

[33] W. Yan, R. Woodard and D. Sornette, Phys. A 391, 1361 (2012).

[34] D. Sornette and A. Helmstetter, Phys. A, 318, 577-591 (2003)

[35] G. Pryce and K. Gibb, Real Estate Econ. 34, 377 (2006)

[36] D. R. Cox and D. Oakes, Analysis of survival data. (Chapman and Hall/CRC, Boca Raton London New York Washington D. C., 1984)

[37] J. Y. Campbell, A. Lo and J. A. C. MacKinlay, The econometrics of financial time series.. (Princeton University Press, Princeton, 1997).

[38] J. Fry, J. of Applied Res. in Financ. 2, 131 (2010).

[39] D. MacKenzie and T. Spears, 'The formula that killed Wall Street?' The Gaussian Copula and the material cultures of modelling. (Working Paper, The University of Edinburgh, 2012).

[40] L. Borland, Quant. Financ. 12, 1367 (2012).

[41] J. Coaffee, Terrorism, risk and the city. (Ashgate, Aldershot, 2003).

[42] N. Carnot, V. Koen, and B. Tissot, Economic forecasting and policy, 2nd edn. (Palgrave Macmillan, Basingstoke New York, 2011).

[43] T. Plummer, Forecasting financial markets. The psychology of successful investing. (Kogan Page, London, 2006).

[44] D. Hillier, S., Ross, R., Westerfeld, J., Jaffe, and B. Jordan, Corporate Finance, 4th edn. (McGraw-Hill, Maidenhead, Berkshire, 2010). 